\title{
Nonlinear vortex lattice method for stall prediction
}

\author{
Hasier Goitia ${ }^{1 *}$, Raúl Llamas ${ }^{2}$ \\ ${ }^{1}$ Airbus, Loads \& Aeroelastics, 316 Route de Bayonne, 31060 Toulouse, France \\ ${ }^{2}$ Airbus, Future Projects Office, Paseo John Lennon S/N, 28906 Getafe, Spain
}

\begin{abstract}
The stall behavior of an empennage is a crucial and conditioning factor for its design. Thus, the preliminary design of empennages requires a fast low-order method which reliably computes the stall behavior and which must be sensitive to the design parameters (taper, sweep, dihedral, airfoil, etc.). Handbook or semi-empirical methods typically have a narrow scope and low fidelity, so a more general and unbiased method is desired. This paper presents a nonlinear vortex lattice method (VLM) for the stall prediction of generic fuselage-empennage configurations which is able to compute complete aerodynamic polars up to and beyond stall. The method is a generalized form of the van Dam algorithm, which couples the potential VLM solution with $2.5 \mathrm{D}$ viscous data. A novel method for computing $2.5 \mathrm{D}$ polars from 2D polars is presented, which extends the traditional infinite swept wing theory to finite wings, relying minimally on empirical data. The method has been compared to CFD and WTT results, showing a satisfactory degree of accuracy for the preliminary design of empennages.
\end{abstract}

\section{Introduction}

The core of the methodology is a vortex lattice method coupled with viscous aerodynamic polars. This is achieved with a generalized version of the $\alpha$-method presented by van Dam [1]. The vortex lattice method implementation employed is described in section 2. The viscous coupling algorithm is presented in section 3 , under the assumption that the section polars are available. Section 4 presents a methodology for computing section polars from 2D polars. Finally, some results obtained with the present method are shown in section 5.

This project has been supported entirely by the Clean Sky 2 program.

\section{Vortex lattice method}

There are several possible implementations of vortex lattice methods (VLM). Katz and Plotkin [2] present a thorough explanation of the method. For this implementation, a strip method with horseshoe elements is chosen. As the chordwise information will be provided by the viscous polars, there is no benefit to increasing the number of chordwise panels.

A wing is described by its origin position and a set of parameters as a function of its local spanwise coordinate $\bar{y}$, namely the chord $c(\bar{y})$, the $x$ and $z$ offset $x_{o f f}(\bar{y})$ and $z_{o f f}(\bar{y})$, the

\footnotetext{
* Corresponding author: hasier.goitia-hernandez@airbus.com
} 
geometric twist $\theta(\bar{y})$, and the local airfoil. The wing is discretized into $N$ spanwise panels. The discrete geometrical parameters are taken from the center of the panel in the local $\bar{y}$ direction. For each panel, the relevant local parameters are the chord $c_{i}$, the dihedral $\phi_{i}$, the geometric quarter-chord sweep $\Lambda_{i}^{\text {geom }}$, and the twist $\theta_{i}$. The normal vector of each panel $\boldsymbol{n}_{\boldsymbol{i}}$ is computed and a control point $C P_{i}$ is placed at the $3 c / 4$ line, in the middle of the panel, as shown in Figure 1.

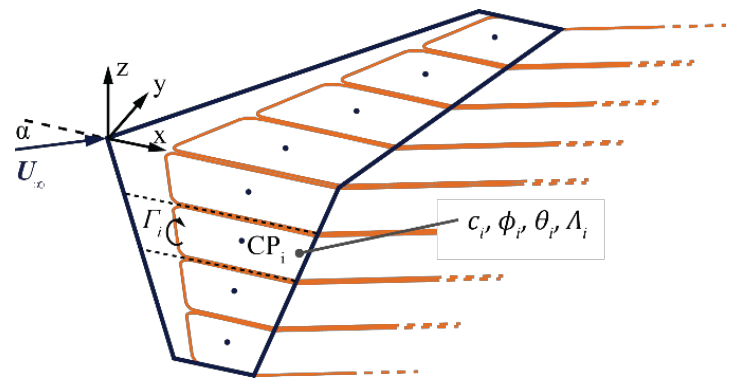

Fig. 1. Diagram of the vortex lattice method with 5-segment horseshoe vortices as the singularity elements. The legs of the HSVs lie on the wing surface and, outside of the wing, in the direction of the free-stream velocity.

Horseshoe vortices (HSV) are used as singularity elements. The HSV head is placed at the quarter-chord line, and each leg extends chordwise downstream over the wing, then in the direction of the free stream towards infinity (Figure 1). Thus, the effect of a HSV can be computed as the sum of five vortex filament segments. The solution for a vortex filament, as well as a code subroutine, can be found in Katz and Plotkin [3].

For a flight condition given by the angles of attack and sideslip $\alpha$ and $\beta$, the free-stream velocity is

$$
\boldsymbol{U}_{\infty}=U_{\infty}(\cos \alpha \cos \beta \boldsymbol{i}+\cos \alpha \sin \beta \boldsymbol{j}+\sin \alpha \boldsymbol{k})
$$

The velocity induced by the HSV of panel $n$ on the CP of panel $m$ is $\boldsymbol{v}_{\boldsymbol{m}, \boldsymbol{n}}$. Since the intensity of the HSVs at this stage is unknown, it is useful to use $\overline{\boldsymbol{v}}_{\boldsymbol{m}, \boldsymbol{n}}=\boldsymbol{v}_{\boldsymbol{m}, \boldsymbol{n}} / \Gamma_{n}$. The total velocity on the $\mathrm{CP}$ of panel $m$ is

$$
\boldsymbol{U}_{\boldsymbol{m}}=\sum_{n=1}^{N} \overline{\boldsymbol{v}}_{\boldsymbol{m}, \boldsymbol{n}} \Gamma_{n}+\boldsymbol{U}_{\infty}
$$

The Neumann boundary conditions arise from the fact that the flow cannot penetrate the wing surface, so a zero normal velocity is imposed at the CPs, $\boldsymbol{U}_{m} \cdot \boldsymbol{n}_{m}=0$, yielding:

$$
\sum_{n=1}^{N}\left(\overline{\boldsymbol{v}}_{m, n} \cdot \boldsymbol{n}_{m}\right) \Gamma_{n}=-\boldsymbol{U}_{\infty} \cdot \boldsymbol{n}_{m}
$$

Writing equation 3 for each panel yields a linear system of $N$ equations where the unknowns are the HSV intensities. The system can be written in matrix form as $[A I C][\Gamma]=$ $[B]$, where the RHS column vector $[B]$ contains the terms $-\boldsymbol{U}_{\infty} \cdot \boldsymbol{n}_{\boldsymbol{m}}$ and is a function of the flow angles $\alpha$ and $\beta$ seen by each panel. The HSV circulations are computed solving the linear system, and the $C_{l}$ of each panel is found with the Kutta-Joukowski theorem.

$$
\begin{gathered}
{[\Gamma]=[A I C]^{-1}[B]} \\
L_{i}^{\prime}=\rho U_{\infty} \Gamma_{i}=\frac{1}{2} \rho U_{\infty}^{2} c_{i} C_{l_{i}} \rightarrow C_{l_{i}}=\frac{2 \Gamma_{i}}{c_{i} U_{\infty}}
\end{gathered}
$$




\section{Viscous coupling}

The VLM can be coupled with 2.5D viscous polars to model the nonlinear behavior of the wing. In this section, it will be assumed that the $2.5 \mathrm{D}$ polars for each wing section are available (see section 4 for a method for computing $2.5 \mathrm{D}$ polars from the airfoil geometry). In practice, the $2.5 \mathrm{D}$ polars are computed for certain key sections (such as the root and the tip) and the rest are linearly interpolated. The coupling is achieved by introducing a local angle of attack correction for each panel $\Delta \alpha_{i}^{\prime}$, such that the flow angles seen by each panel are modified. This alters the $[B]$ vector in equation 6 and, consequently, the HSV intensities $[\Gamma]$ and $C_{l}$ distribution. The flow angles $\alpha$ and $\beta$ seen by a given panel $i$ are related to the local angle of attack $\alpha_{i}^{\prime}$ and the local dihedral $\phi_{i}$ through:

$$
\begin{aligned}
\alpha_{i} & =\alpha_{i}^{\prime} \cos \phi_{i} \\
\beta_{i} & =\alpha_{i}^{\prime} \sin \phi_{i}
\end{aligned}
$$

The modified van Dam algorithm is as follows (where the subindex $i$ indicates that the computation must be done for each panel):

a) Set $\Delta \alpha_{i}=0$

b)Find the corrected flow angles $\alpha_{i}^{*}$ and $\beta_{i}^{*}$, and compute $[B]$ with the corrected angles:

$$
\begin{gathered}
\alpha_{i}^{*}=\alpha-\left(\Delta \alpha_{i}+\alpha_{0_{i}}\right) \cos \phi_{i} \\
\beta_{i}^{*}=\beta-\left(\Delta \alpha_{i}+\alpha_{0_{i}}\right) \sin \phi_{i}
\end{gathered}
$$

c) Solve the VLM. The resulting lift coefficient of each panel is $C_{l_{V L M}}$.

d)Find the effective and induced angles of attack of each strip:

$$
\alpha_{\text {ind }_{i}}=\alpha \cos \phi_{i}+\beta \sin \phi_{i}-\left(\frac{C_{l_{V L M_{i}}}}{2 \pi}+\alpha_{0_{i}}+\Delta \alpha_{i}^{\prime}\right)-\Delta \alpha_{i}^{\prime}+\theta_{i}
$$

e) Interpolate the $\alpha_{l o c_{i}}$ in the 2.5D polar of the corresponding panel to find $C_{l_{e f f_{i}}}$ and $C_{d_{\text {eff }}}$.

f) Perform an axes transformation to find the lift and drag coefficients in the local flow axes.

$$
C_{l_{\text {visc }_{i}}}=C_{l_{\text {eff }}} \cos \alpha_{\text {ind }_{i}}-C_{d_{\text {eff }} \text { in }} \sin \alpha_{\text {ind }_{i}}
$$

g) Update the value of $\Delta \alpha_{i}$. The introduction of a smoothing factor $\zeta$ is not strictly necessary but beneficial in practice to aid numerical convergence.

$$
\Delta \alpha_{i} \leftarrow \Delta \alpha_{i}+\frac{C_{l_{V L M_{i}}}-C_{l_{v i s c_{i}}}}{2 \pi} \cdot \zeta
$$

h)Find the maximum error from all panels:

$$
\text { err }=\max \left(\left|C_{l_{V L M_{i}}}-C_{l_{\text {visc }}}\right|\right)
$$

i) If the error is below a certain tolerance $\varepsilon$, take $C_{l_{V L M_{i}}}$ as the converged value for each panel; otherwise, repeat from step b).

\section{Aerodynamic polars: 2D to 2.5D}

The main difficulty of the described method is computing the $2.5 \mathrm{D}$ polars. They can be can be obtained from CFD results or wind-tunnel tests, though when the results of such costand time-extensive methods are available, the present method is evidently redundant. On the other hand, 2D polars can be easily computed with a number of airfoil solvers, such as XFOIL [4], MSES [5] or 2D CFD codes. This section presents a novel method for transforming the $2 \mathrm{D}$ polars into the $2.5 \mathrm{D}$ polars given the wing geometry. 
The difference between the $2.5 \mathrm{D}$ and $2 \mathrm{D}$ polars arises from the sweep, which produces a spanwise boundary layer flow (from root to tip if the sweep is backwards, as shown in Figure 2 ). The main consequence of this is that the $\mathrm{BL}$ is thinner at the root (higher effective Reynolds, delaying stall and achieving a higher $C_{l_{\max }}$ ) and thicker at the tip (having the opposite effect). Thus, backwards sweep promotes stall from the tip, and forward sweep promotes stall from the root, as can be seen in the tuft studies from NACA TN 2445 [6].

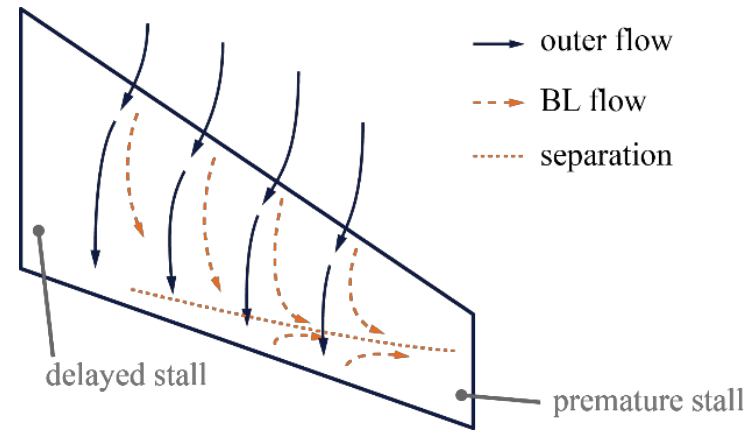

Fig. 2. Diagram of outer and boundary-layer flow over a backwards-swept wing.

In the general case of arbitrarily large sideslip and dihedral angles, calculating the sweep angle of a wing or wing section becomes nontrivial. The local sweep of a wing section is a factor of both the wing geometry -a combination of geometrical sweep and dihedral-and the flow angles. The relevant sweep angle considered here is that formed between the projection of $\boldsymbol{U}_{\infty}$ onto the panel surface and the perpendicular to the quarter-chord line. The projection of a generic vector $\boldsymbol{v}$ onto a plane defined by its normal vector $\boldsymbol{n}$ can be written as:

$$
v_{t}=v-v_{n}=v-(v \cdot n) n
$$

where $\boldsymbol{v}_{t}$ and $\boldsymbol{v}_{n}$ are the tangential and normal components of $\boldsymbol{v}$ with respect to the plane.

Thus, the projection of the free-stream velocity $\boldsymbol{U}_{\infty}$ onto the $i^{\text {th }}$ panel with normal vector $\boldsymbol{n}_{i}$ is

$$
\boldsymbol{U}_{\infty \boldsymbol{i}}^{\boldsymbol{t}}=\boldsymbol{U}_{\infty}-\left(\boldsymbol{U}_{\infty} \cdot \boldsymbol{n}_{i}\right) \boldsymbol{n}_{i}
$$

Using the local $x^{*}$ axis unitary vector $\boldsymbol{i}^{*}$, the local sweep seen by panel $i$ is (Figure $3 \mathrm{a}$ )

$$
\Lambda_{\text {loc } i_{i}}=\Lambda_{\text {geom }_{i}}+\Lambda_{i}^{*}=\Lambda_{\text {geom }_{i}}+\cos ^{-1}\left(\frac{\left\|\boldsymbol{U}_{\infty_{i}}^{t}\right\|}{\boldsymbol{U}_{\infty_{i}}^{t} \cdot \boldsymbol{i}^{*}}\right)
$$

In the case of an infinite swept wing, the 2D airfoil polar can be transformed into the wing section 2.5D polar with the classical sweep theory, as shown in the ESDU methodology [7]. Mariens et al. [8] present a method for the transformation of $C_{D}$ due to sweep. In practice, and especially at low Mach numbers, some simplifications can be taken, which greatly speed up the computation process without significantly compromising the results.

Given an infinite-span wing of constant sweep $\Lambda$ flying at Mach number $M$ and Reynolds number $R e$, the proposed simplified methodology is the following:

a) Extract the airfoil geometry from the wing section.

b) Compute the airfoil polar with a 2D aerodynamic solver at Mach number $M$ and Reynolds number $R e$, obtaining $C_{l_{2 D}}\left(\alpha_{2 D}\right)$ and $C_{d_{2 D}}\left(\alpha_{2 D}\right)$.

c) The $2.5 \mathrm{D}$ polar is

$$
\begin{gathered}
C_{l_{2.5 D}}=C_{l_{2 D}} \cos ^{2} \Lambda \\
\alpha_{2.5 D}=\left(\alpha_{2 D}-\alpha_{0}\right) \cos \Lambda+\alpha_{0}
\end{gathered}
$$

where $\alpha_{0}$ the zero-lift angle of attack of the 2D polar. 
The effect of wing sweep, as well as the correlation with the simplified methodology, is shown in Figure 3b. It can be observed that, despite the errors in the original XFOIL polar (especially in the negative stall region) the proposed transformation is adequate.

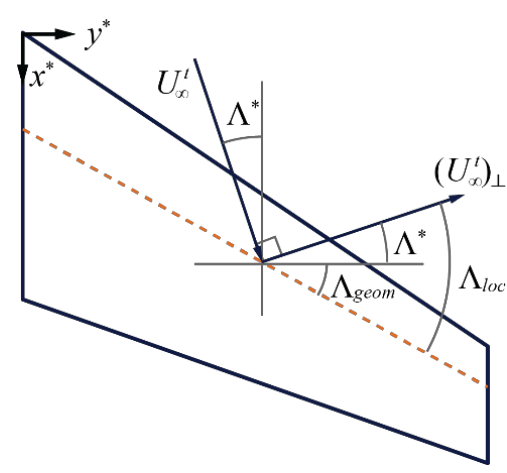

(a)

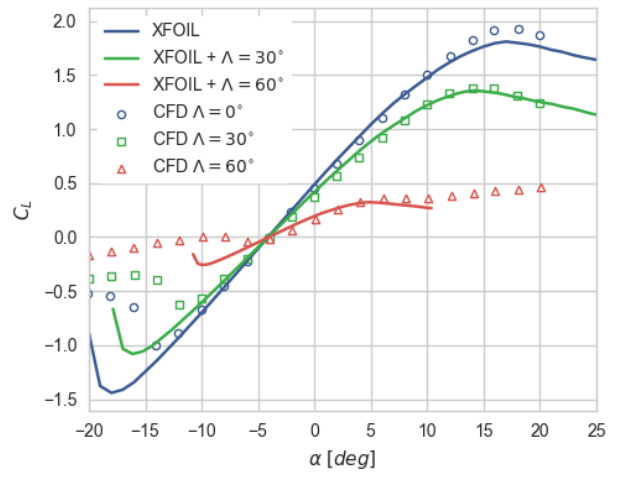

(b)

Fig. 3. (a) Relevant angles related to sweep in a generic wing in a free stream of velocity $\boldsymbol{U}_{\infty}$, in the local coordinates of the wing plane. (b) Comparison of 2.5D polar for an infinite wing with a NACA 4416 profile and sweeps $\Lambda=0^{\circ}, \Lambda=30^{\circ}$ and $\Lambda=60^{\circ}$, at $M=0.2$ and $R e=6 \cdot 10^{6}$ as computed by CFD (TAU2D) and with the described methodology starting from a 2D polar computed with XFOIL.

In a finite swept wing, the $2.5 \mathrm{D}$ polars vary along the span, having a higher $C_{l_{\max }}$ at the root than at the tip. This behavior is discussed in a paper by Hosangadi et al. [9] using the CFD results from [10]. We propose a method for modelling the $2.5 \mathrm{D}$ polars of finite wings based on assigning an effective (i.e. artificial) sweep to each spanwise section, then applying the sweep correction described in section 4 . The effective sweep for the $i^{\text {th }}$ panel is

$$
\Lambda_{e f f_{i}}=\Lambda_{l o c_{i}} \cdot \kappa_{\Lambda_{i}}
$$

where the effective sweep correction $\kappa_{\Lambda_{\mathrm{i}}}$ is hypothesized to be a function of only the spanwise position and aspect ratio. To account for the possibility of an increase in $C_{l_{\max }}$ as occurs at the wing root, equation 26 is replaced by

$$
\begin{gathered}
C_{l_{2.5 D}}=C_{l_{2 D}} F_{\Lambda}^{2} \\
\alpha_{2.5 D}=\left(\alpha_{2 D}-\alpha_{0}\right) F_{\Lambda}+\alpha_{0}
\end{gathered}
$$

where

$$
F_{\Lambda}=\left\{\begin{array}{rr}
2-\cos \Lambda_{e f f}, & \Lambda_{e f f}<0 \\
\cos \Lambda_{e f f}, & \Lambda_{e f f} \geq 0
\end{array}\right.
$$

A small set of wings of various aspect ratios and sweep angles, including those presented in Hosangadi et al. [9], has been studied. The spanwise distribution of the $C_{l_{\max }}$ of these wings indicate that the hypothesized $\kappa_{\Lambda}$ function does indeed exist. Assuming that the effect of the root and the tip are independent and can be superimposed, the proposed sweep correction function is modelled as

$$
\kappa_{\Lambda}(y)=1+\kappa_{\text {root }}\left(\eta_{\text {root }}^{\prime}\right)+\kappa_{\text {tip }}\left(\eta_{\text {tip }}^{\prime}\right)
$$

where $\eta_{\text {root }}^{\prime}$ and $\eta_{\text {tip }}^{\prime}$ are nondimensional spanwise positions normalized with the mean geometric chord. They are defined as

$$
\begin{gathered}
\eta_{\text {root }}^{\prime}(y)=\frac{y-y_{\text {root }}}{\bar{c}}=A R\left(\eta(y)-\eta_{\text {root }}\right)=A R \eta \\
\eta_{\text {tip }}^{\prime}(y)=\frac{y-y_{\text {tip }}}{\bar{c}}=A R\left(\eta(y)-\eta_{\text {tip }}\right)=A R(\eta-1)
\end{gathered}
$$


where $\eta$ is the standard nondimentional spanwise position $2 y / b$. The variables $\eta_{\text {root }}^{\prime}$ and $\eta_{\text {tip }}^{\prime}$ measure how many chords away a spanwise position is from the root or the tip. The functions $\kappa_{\text {root }}\left(\eta_{\text {root }}^{\prime}\right)$ and $\kappa_{\text {tip }}\left(\eta_{\text {tip }}^{\prime}\right)$ (Figure $\left.4 a\right)$ have been designed with Bézier curves to best fit the data gathered from the aforementioned set of wings. The resulting $\kappa_{\Lambda}(\eta)$ function is plotted in Figure $4 \mathrm{~b}$ for several aspect ratios. Note that for large aspect ratios, the solution for the infinite swept wing $\left(\kappa_{\Lambda}=1\right)$ is recovered for an area in the middle of the wing. The formulation of $\kappa_{\Lambda}$ is valid for approximately $A R>5$; for smaller aspect ratios, the effect of the root overpowers that of the tip and the result is nonphysical.

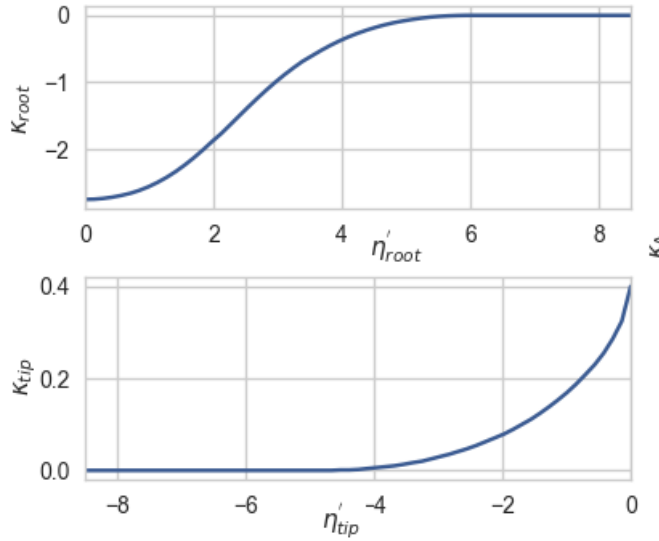

(a)

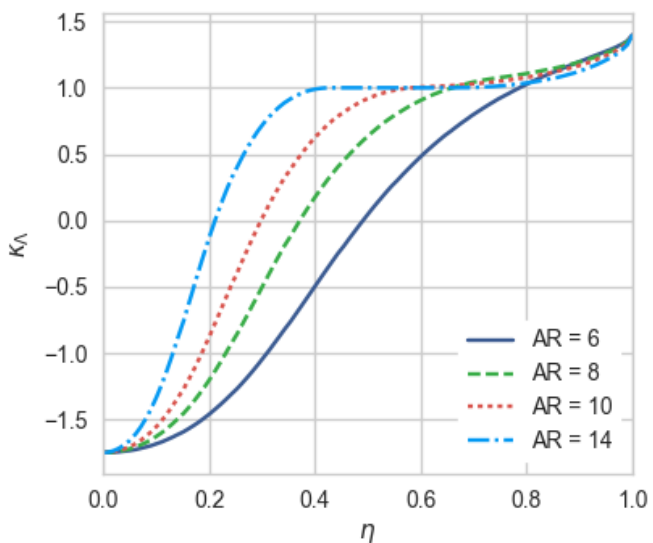

(b)

Fig. 4. (a) Values of $\kappa_{\text {root }}$ and $\kappa_{\text {tip }}$ as a function of the nondimensional parameters $\eta_{\text {root }}^{\prime}$ and $\eta_{\text {tip }}^{\prime}$, respectively. (b) Effective sweep correction function $\kappa_{\Lambda}$ as a function of the nondimensional spanwise position $\eta=2 y / b$, for various aspect ratios.

\section{Results}

The lift curves of the set of wings from Hosangadi et al. [9] as computed by CFD and the present method are shown in Figure 5a. It may be observed that the present method has the proper sensitivity to the wing sweep. Figure $5 \mathrm{~b}$ shows the comparison between the present method and NACA wind tunnel tests for a swept wing of $A R=8$. The result for the van Dam $\alpha$-method using 2D polars has also been plotted, to demonstrate the effect of the sweep correction function $\kappa_{\Lambda}$. Figure $5 \mathrm{c}$ presents the lift curve for an isolated wing with a geometry which is representative of a modern HTP. It is compared to CFD results from the Selena project [12]. The correlation between the present method and the CFD results are good, which suggests the validity of the sweep correction function $\kappa_{\Lambda}$ for aspect ratios as low as $A R=5$.

In the scope of aircraft design, the interest is generally in the analysis of wing-fuselage or tail-fuselage geometries, rather than isolated lifting surfaces. Our proposed method for accounting for the effect of the fuselage on the tailplane consists on modelling the fuselage as a cylindrical surface of vortex rings, removing all internal wing panels. The cylindrical surface must extend a length of at least $\sim 3 \bar{c}$ in front and behind the lifting surface to avoid unphysical effects. The following results use the present method to replicate a CFD analysis of a body-tail geometry representative of a modern airliner. Figure 6 a shows the VLM representation of the geometry. The spanwise loading is shown in Figure $6 \mathrm{~b}$. The present method correlated well to the CFD result. The 3-dimensional lift curve of the body-tail configuration presented in Figure 5d shows an acceptable correlation of the present method to wind tunnel tests and CFD computations. This indicates a strong potential of the method for analyzing complex configurations and interactions between lifting surfaces and bodies. 


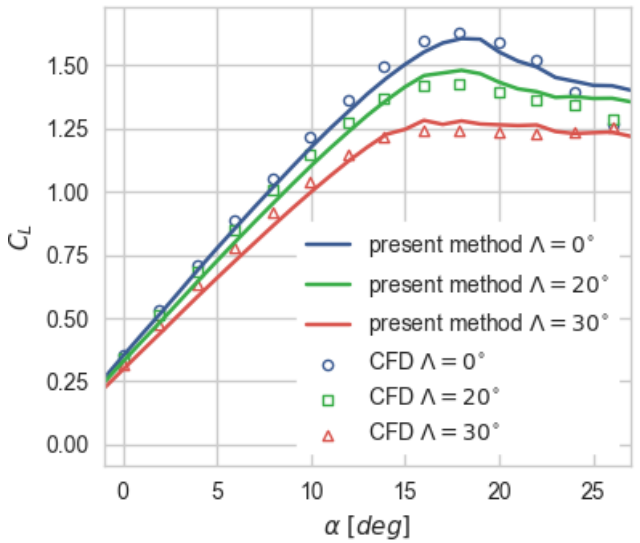

(a)

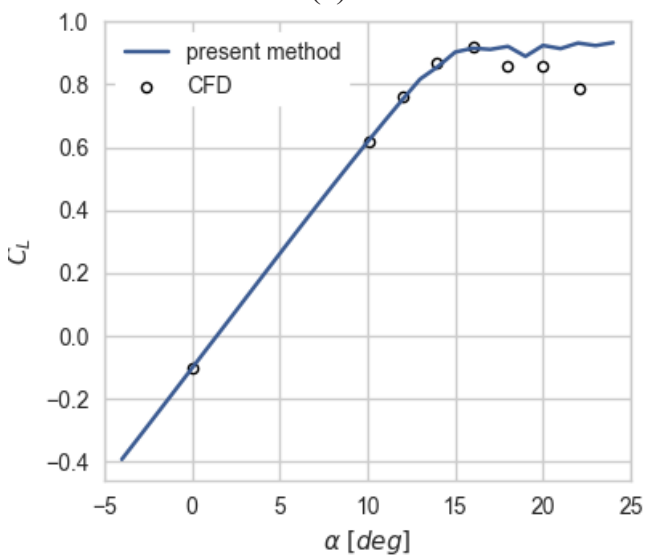

(c)

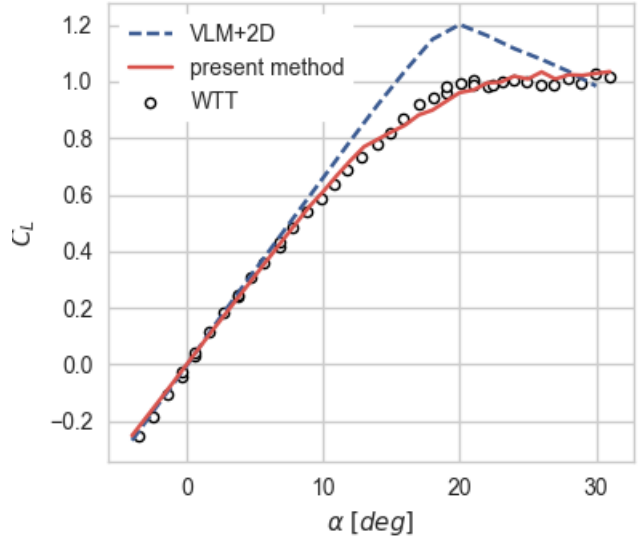

(b)

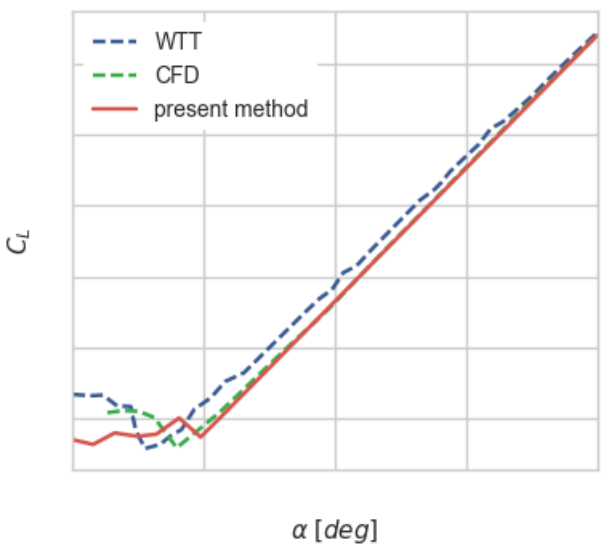

(d)

Fig. 5. Comparison of $C_{L}-\alpha$ as computed by CFD or wind tunnel testing and the present method, for (a) three wings from [10] $\left(A R=12, T R=1\right.$, NACA 4416 profiles, $\left.R e=3 \cdot 10^{6}, M=0.2\right)$, (b) the NACA TR1208 wing [11] $\left(A R=8, \lambda=0.45, \Lambda=45^{\circ}\right.$, NACA $63{ }_{1} \mathrm{~A} 012$ profiles, $\left.R e=4 \cdot 10^{6}\right)$, (c) the Selena project geometry ( $\left.A R=5, \lambda=0.35, \Lambda=28^{\circ}, R e=4 \cdot 10^{6}, M=0.2\right)$, and (d) the body-tail configuration.

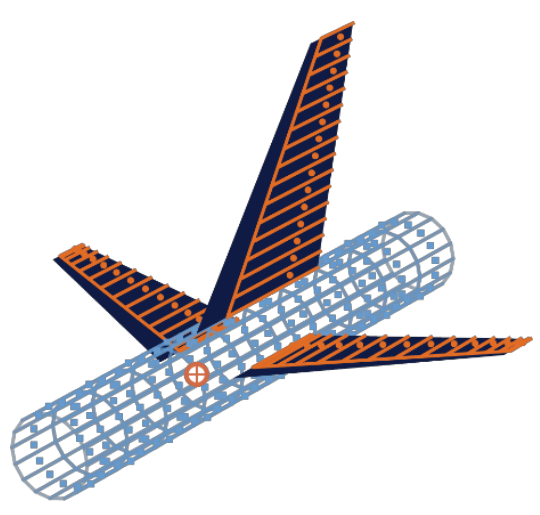

(a)

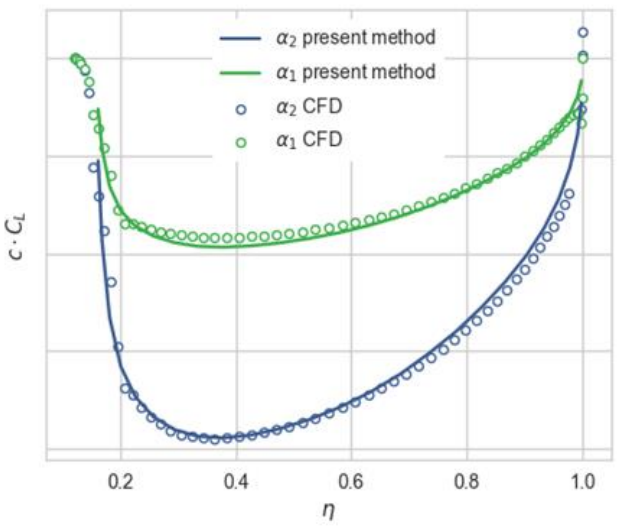

(b)

Fig. 6. (a) VLM model of a conventional empennage and fuselage rear end. (b) Spanwise aerodynamic loading on the exposed HTP surface, at two different angles of attack in the linear region. 


\section{Conclusions and Way Forward}

The present method has been implemented as a Python library with a simple user interface which enables the user to generate wing and fuselage geometries, read 2D input polars from XFOIL, MSES and Tau2D, and compute complete 3-dimensional lift curves. Typical computation times for an isolated wing are $\sim 4 s$ ( $\sim 25 s$ for a wing-fuselage configuration) on a single core. Given this low computational cost, the present method has great potential for use in the preliminary design process. The comparison cases shown in this report suggest that the method is able to predict the onset of stall with reasonable accuracy for a wide design space of isolated wings and wing-body configurations with proper sensitivity to the design parameters. Notably, the method is predominantly physically-based, scarcely relying on empirical data. Only the $\kappa_{\Lambda}$ function -which is indirectly a loose model for the evolution of the boundary layer thickness due to sweep- relies on empirical data. A full validation of the method is still pending. In order to achieve this, an extensive set of wind tunnel test results of a large design space of wing geometries are required. Moreover, the cases for which the $3 \mathrm{D}$ stall behavior cannot be derived by augmenting the $2 \mathrm{D}$ data must still be identified to find the limits of applicability of the present method.

\section{References}

[1] C. P. van Dam, "The aerodynamic design of multi-element high-lift systems for transport airplanes," Progress in Aerospace Sciences, vol. 38, pp. 10-144, 2002.

[2] J. Katz and A. Plotkin, "Three-Dimensional Numerical Solutions," in Low-Speed Aerodynamics. From Wing Theory to Panel Methods, New York, Cambridge University Press, 2001, pp. 331368.

[3] J. Katz and A. Plotkin, "Singularity Elements and Influence Coefficients," in Low-Speed Aerodynamics. From Wing Theory to Panel Methods, New York, Cambridge University Press, 2001, pp. 230-261.

[4] M. Drela, "XFOIL: An Analysis and Design System for Low Reynolds Number Airfoils," in Low Reynolds Number Airfoil Aerodynamics, University of Notre Dame, June 1989.

[5] M. Drela, A User's Guide to MSES 3.05, Massachusetts Institute of Technology. Department of Aeronautics and Astronautics, July 2007.

[6] P. E. Purser and M. L. Spearman, "NACA TN 2445: Wind-Tunnel Tests at Low Speed of Swept and Yawed Wings Having Various Plan Forms," National Advisory Committee for Aeronautics, Washington, December 1951.

[7] ESDU, "Data Item 90008: Transonic aerodynamics of aerofoils and wings," IHS ESDU, April 1990.

[8] J. Mariens, A. Elham and M. Van Tooren, "Quasi-Three-Dimensional Aerodynamic Solver for Multidisciplinary Design Optimization of Lifting Surfaces," Journal of Aircraft, vol. 51, no. 2, pp. 547-558, May 2014.

[9] P. Hosangadi, R. Paul and A. Gopalarathnam, "Improved Stall Predictions for Swept Wings Using Low-Order Aerodynamics," in AIAA, June 2015.

[10] J. Petrilli, R. Paul, A. Gopalarathnam and N. T. Frink, "A CFD Database for Airfoils and Wings at Post-Stall Angles of Attack," in AIAA, 2013.

[11] W. C. Schneider, "Technical Report 1208: A comparison of the spanwise loading calculated by various methods with experimental loadings obtained on a 45 degree sweptback wing of aspect ratio 8.02 at Reynolds number of 4.0x10(6)," NACA, 1951.

[12] M. Chávez-Modena, E. Ferrer, J. Martínez and J. Cabello, "Industrial benchmark simulations of detached flows using XFlow," in EUCASS, Madrid, 2019.

[13] J. C. Sivells and R. H. Neely, "NACA Technical Note No. 1269," National Advisory Commitee for Aeronautics, Washington, 1947. 